\title{
What influences prescribing decisions in a multimorbidity and polypharmacy context on the acute medical unit? An interprofessional, qualitative study
}

\author{
Peter Rivers ${ }^{1}$, Nigel Langford ${ }^{2}$, Andrew Whitehead $^{3}$, and Tim Harrison ${ }^{1}$ \\ ${ }^{1}$ De Montfort University - City Campus \\ ${ }^{2}$ University Hospitals of Leicester NHS Trust \\ ${ }^{3}$ Buckinghamshire Healthcare NHS Trust
}

July 28, 2020

\begin{abstract}
ABSTRACT Rationale, aims and objectives The primary aim of the study was to understand the mindset of doctors and pharmacists, as they embark upon prescribing in a polypharmacy and multi-morbidity context during routine practice at a hospital acute admissions unit. The study also aimed to evaluate to what extent attitudes, embedded within real-life decisionmaking scenarios, relate to existing theory and models of prescribing decisions. Methods Anonymised case studies were identified from the medical notes of patients aged 65 and over with conditions likely to be associated with multi-morbidity, medication issues and polypharmacy: namely: fall, urinary tract infection, confusion or lower respiratory tract infection. A total of 39 doctors based on the acute medical admissions unit and 9 pharmacists were recruited to one of three focus groups. Patient casestudies provided the context for discussion from which verbatim transcripts were thematically analysed using an interpretative, qualitative approach. Sub-themes were matched to Murshid and Mohaidin's proposed model of physician prescribing decisions. Results Seven principal themes were identified that were associated with prescribing decisions on the acute medical unit, namely, 'patient characteristics', 'drug characteristics', 'pharmacist factors', 'trustworthiness', 'reliability of medication history, 'competing pressures and priorities' and 'responsibilities of prescribers'. Conclusion Prescribing decisions on the acute medical admissions unit were influenced by a variety of factors, some of which have already been acknowledged within existing theories and models. The findings provisionally offer new insights, which, subject to confirmation by further research, bring to light three attitudinal characteristics that may impact negatively upon the quality of prescribing decisions. These include, first, how perceived poor reliability of medication history may result in information gaps that compromise prescribing decisions; second, how competing pressures and priorities restrict doctors' aptitude to conduct a review of medication and finally, how doctors may rationalise the assignment of medication review to the GP.
\end{abstract}

\section{TITLE PAGE}

Title

What influences prescribing decisions in a multimorbidity and polypharmacy context on the acute medical unit? An interprofessional, qualitative study

\section{Short running title}

Prescribing decisions on the acute medical unit

Author names and affiliations

Dr Peter H Rivers, Honorary Reader, School of Pharmacy, Hawthorn Building, De Montfort University, The Gateway, Leicester LE1 9BH ${ }^{+}$. Tel: 01332 676241. Email: peterrivers@btinternet.com 
Dr Nigel Langford, Email: nigellangford@nhs.net. Honorary Senior Lecturer, University of Leicester, Consultant Clinical Pharmacologist and Acute and General Physician

Dr Andrew Whitehead, Specialty Registrar, Intensive Care Medicine and Anaesthesia, Buckinghamshire Healthcare NHS Trust. Email: apwhitehead@doctors.org.uk

Mr Tim Harrison, Associate Professor, School of Pharmacy, De Montfort University Email: tharrison@dmu.ac.uk.

+ Corresponding author's present address: 17 Cole Lane, Ockbrook, Derby DE72 3RD

Correspondence to : Dr Peter Rivers: peterrivers@btinternet.com

Running title: Prescribing decisions on the acute medical unit

KEY WORDS

multimorbidity, polypharmacy, prescribing

WORD COUNT:4862 (excluding abstract, key words and references) ABSTRACT

Rationale, aims and objectives

The primary aim of the study was to understand the mindset of doctors and pharmacists, as they embark upon prescribing in a multimorbidity and polypharmacy context during routine practice at a hospital acute admissions unit. The study also aimed to evaluate to what extent attitudes, embedded within real-life decision-making scenarios, relate to existing theory and models of prescribing decisions.

\section{Methods}

Anonymised case studies were identified from the medical notes of patients aged 65 and over with conditions likely to be associated with multimorbidity, medication issues and polypharmacy: namely: fall, urinary tract infection, confusion or lower respiratory tract infection. A total of 39 doctors based on the acute medical admissions unit and 9 pharmacists were recruited to one of three focus groups. Patient case-studies provided the context for discussion from which verbatim transcripts were thematically analysed using an interpretative, qualitative approach. Sub-themes were matched to Murshid and Mohaidin's proposed model of physician prescribing decisions.

\section{Results}

Seven principal themes were identified that were associated with prescribing decisions on the acute medical unit, namely, 'patient characteristics', 'drug characteristics', 'pharmacist factors', 'trustworthiness', 'reliability of medication history, ' competing pressures and priorities' and 'responsibilities of prescribers'.

\section{Conclusion}

Prescribing decisions on the acute medical admissions unit were influenced by a variety of factors, some of which have already been acknowledged within existing theories and models. The findings provisionally offer new insights, which, subject to confirmation by further research, bring to light three attitudinal characteristics that may impact negatively upon the quality of prescribing decisions. These include, first, how perceived poor reliability of medication history may result in information gaps that compromise prescribing decisions; second, how competing pressures and priorities restrict doctors' aptitude to conduct a review of medication and finally, how doctors may rationalise the assignment of medication review to the GP.

\section{INTRODUCTION}

It is widely recognised that the more medicines patients take, the greater the risk of adverse drug reactions and hospitalisation. ${ }^{1}$ Indeed, prescription medication is implicated as a causal factor in approximately $7 \%$ of hospital admissions. ${ }^{2}$ However, when treating patients with multiple morbidities, deciding which of several 
medicines are appropriate ('appropriate polypharmacy') and which are not ('problematic polypharmacy') ${ }^{3}$ represents a prescribing decision dilemma, that, to date, remains largely unresolved especially when multiple prescribers focus upon different disease processes.

A comprehensive review of theories and models of prescribing decisions by Murshid and Mohsen describes a wide range of variables that relate to physicians' decisions to prescribe medicines. ${ }^{4}$ The theories of persuasion, planned behaviour and agency theory have enhanced our understanding of how prescribing decisions may be influenced by the characteristics of patients, (e.g. patient expectations), pharmaceutical marketing ${ }^{5}$, the characteristics of drugs, the ratio of drug cost to benefit and physician habit persistence. Social power theory is also important in terms of understanding how pharmacist - physician collaboration and the level of trustworthiness between health care professionals may influence prescribing decisions. However, in our experience, this knowledge has not yet been consistently translated into practice to ensure problematic polypharmacy is avoided.

There is no shortage of professional guidance on how best to encourage appropriate prescribing in the UK in the face of polypharmacy. ${ }^{3} 7891011$ Indeed, prescribing guidance tools such as Beers criteria for the elderly ${ }^{12}$ and STOPP/START ${ }^{13}$, are readily available. However, a recent randomised controlled trial carried out in six European hospital medical centres reported poor uptake by clinicians of SENATOR software-generated medication advice based upon STOPP/START prescribing rules. ${ }^{14}$ A shared aim of all polypharmacy guidance is to recognise the patient as an individual who often has multiple problems, rather than as a series of individual conditions where multiple individual clinical guidelines may be applied. Thus, some goals may need to be modified to enable the overall health of an individual to be optimised whilst ultimately ensuring prescribed medicines are safe.

Owing to the on-going conflict between problematic polypharmacy and patients' presentation to hospital with multimorbidity, we decided to find out whether prescribing decisions are influenced by factors other than those that have already been identified by existing theories and models of prescribing. We, therefore, decided to evaluate the mindset of doctors and pharmacists involved in making prescribing decisions in an acute hospital medical unit (AMU). The term 'mindset' reflects the habitual attitudes and ways of thinking that contribute towards a mental framework within which prescribing decisions are made. The primary aim of the study was to gain an understanding of the mindset of prescribers during routine practice and to evaluate whether this mindset, embedded within real-life decision-making scenarios, relates to existing theory and models of prescribing decisions.

\section{METHODS}

Qualitative data were obtained as part of a wider quantitative study investigating the prescribing history of patients from the time of hospital admission until the day of discharge. Fieldwork was conducted by three cohorts of research assistants who were undertaking their final year of the MPharm pharmacy degree between January and March during 2015, 2016 and 2017. Ethical approval and honorary contracts were granted by UK University Hospitals of Leicester (UHL) and De Montfort University (DMU) for each cohort of students: 2015: UHL Reg. No. 7186e, 2016: DMU FREC 1679, 2017: DMU FREC 1866).

Each year, the research assistants selected a sample of clinical notes of patients who were being treated on the AMU at Leicester Royal Infirmary. The inclusion criteria for selection were: age $65+$ years; admission during the previous week; having one or more of the following presentations, all of which are frequently associated with medication issues and polypharmacy:- fall, urinary tract infection, confusion or lower respiratory tract infection. From this sample, NL selected 6 case studies, of contrasting prescribing complexity, that were judged to be within the experience of participants and who would be expected to manage within their routine practice. These case studies were subsequently shown to medical staff and pharmacists during a focus group in the second phase of the study as a basis for stimulating discussion about prescribing decisions. The median number of medicines prescribed at the point of admission to hospital for patients who were included as case studies was seven ( $\min 2, \max 20)$.

After the quantitative survey had been completed, doctors working within the AMU were invited (by NL 
in his role as AMU consultant physician) to attend a focus group that was held during a normal regular clinical lunchtime meeting located within the hospital premises. Pharmacists were similarly invited to a single separate focus group in 2017. It was made clear that about one hour would be set aside for the focus group and that attendance was voluntary. Those who took part in the study gave written consent after having advance opportunity to read a participant information sheet and a verbal explanation immediately prior to commencement.

A total of forty-eight participants (39 doctors, nine pharmacists) attended the focus groups which included a minimum of nine and a maximum of 19 participants per group. The level of experience of the doctors ranged from the first foundation year up to consultant level. The names of the doctors who attended the focus groups were not recorded. Those who were in their first foundation year attended only one focus group and doctors in more senior grades attended between one and three groups according to their availability to attend. Those who attended the pharmacy focus group were all pharmacists ranging from 'band 6' (newly qualified) to 'band $8 \mathrm{~A}$ ' (specialist pharmacists). A semi-structured topic guide was used by an academic male, non-clinician, facilitator $(\mathrm{PR})$ in order to encourage open and blame-free discussion relating to prescribing decisions arising from the case-studies. During the period of the study PR was employed as pharmacist final project supervisor, researcher, and evaluator at a local school of pharmacy and not previously known by the study participants. The facilitator had extensive previous experience in conducting focus groups in health contexts using unstructured and semi-structured techniques. His background as a pharmacist offered a broad understanding of the clinical use of medicine which facilitated development of rapport with the participants. However, the facilitator endeavoured to remain neutral with respect to his views of prescribing decisions while interacting with the group. Each focus group was also attended by up to three final year undergraduate pharmacy students primarily as non-participant observers although assisting the facilitator by intervening occasionally in order to seek clarification. The number of case studies presented to participants ranged from three to six per focus group depending upon the complexity of the cases and consequently the amount time available. The reason for showing the medical and medication histories was to provide context and to facilitate opening up discussion on prescribing decisions.

Upon commencement of the focus group, participants were invited to reflect upon what they aimed to achieve as prescribers and upon any difficulties that arise when assessing the medication needs of a newly admitted patient. The discussion emanating from these opening questions then provided context for the group to consider prescribing decisions that were shown within the case studies. The facilitator put each case study up on screen and paper copies of the case studies were made available. The facilitator read out, for each case study in turn, the presenting diagnoses and therapeutic actions including medication that was stopped, paused, or started during the period of stay. Then the group was invited to share their thoughts on the case study and to consider whether they would have acted in the same way with respect to prescribing decisions. There were no more set questions as such - the remainder of the focus group was conducted using an unstructured format where questions arose from the ensuing comments and discussion. The facilitator encouraged participants to make their own judgements and comments and, where appropriate, prompted respondents to expand or explain their thinking.

\subsection{Data analysis}

The focus groups were audio-recorded and verbatim transcripts underwent thematic analysis by the authors based upon a pragmatic process where components of experiences were pieced together to form a picture of collective experience of the participants, based upon a method described by Aronson ${ }^{15}$. Coding of the transcripts was initially completed independently and finalised after meetings. The subthemes derived from analysis were matched, where appropriate, to a proposed new model of physician prescribing decisions published by Murshid and Mohaidin ${ }^{4}$.

The initial coding of transcripts followed by aggregation into thematic dimensions and subthemes was informed by our philosophical approach. The study was originally conceived as a descriptive quantitative survey and the focus groups that followed were intended as a means to critique the appropriateness of prescribing decisions as audited against quantitative data derived from the first phase of the study. However, 
it became apparent, after transcription of the three focus groups, that there was greater potential to apply interpretivism in our approach to analysis. We realised that there were further insights to be gained by comprehending the thought processes that provide context and foundation to prescribing decisions. Philosophically, our approach therefore evolved more into alignment with verstehen, a term that was originally introduced by Max Weber ${ }^{16}$ which essentially refers to an understanding of the world as others see it. It is recognised that the term 'interpretivism' embraces a variety of different philosophical approaches. ${ }^{17}$ This study essentially involves human interpretation and we believe that it may be more accurately defined as one that is phenomenologically orientated. Thus, we sought to understand what it is like to be a prescriber and pharmacist on the AMU (their 'lived experience') and to appreciate the conscientiousness of prescribers and prescribing advisors as they embark upon making prescribing decisions. It is important to acknowledge that the medical and pharmacological information that was presented within the case studies, to provide context for participants, was not relevant to this phenomenological analysis because there was no intention to pass judgement over the choice of medicines or to question the appropriateness of decisions. Thus, we felt that it was important to disassociate, from the analysis, clinical details relating to care of patients. For these reasons, the case studies are not included in this paper.

\section{FINDINGS AND DISCUSSION}

The findings highlighted some well-known driving forces that influence prescribing decisions. These include 'patient characteristics', 'drug characteristics', 'drug characteristics, 'pharmacist factors' and 'trustworthiness'. Interestingly, the influence of 'marketing effects' by the pharmaceutical industry, a variable derived from persuasion theory ${ }^{4}$ that has been advocated as a main driver for prescribing decisions in connection with, for example, brands of drug, was not overtly observed in the present study. The reason for this finding is unknown but we postulate that, while complacency must be avoided, there may today be a greater awareness of potential conflict in interest that can arise between pharmaceutical representatives and hospital prescribers. ${ }^{18}$ The findings uncovered three new attitudinal factors that influence prescribing decisions that have not, to date, been described in the literature:1. Reliability of medication history, 2. Competing pressures and priorities and 3. Perceived responsibilities of prescribers. A summary of dimensions and subthemes identified within the findings and presented in relation to existing prescribing theory and models is shown in Table 1.

\subsection{Patient characteristics}

Doctors, in line with the principles of the Hippocratic oath, "use treatment to help the sick ..., but never with a view to injury and wrong-doing", aim to ensure that medication is safe and beneficial. Upon admission to the AMU, the patient's presenting complaint and clinical picture alongside immediate prescribing for acute treatments were primary considerations in the prescribing of medicines. Doctors acknowledged that it was important to ensure that the patient's existing therapy should not be interrupted so long as it continued to be beneficial. The characteristics of the patient, such as confusion, dehydration, or renal function, then modified this decision and acted as a prompt for further prescribing actions. For example, if kidney function was poor, this might prompt a decision to stop, or temporarily withhold, medication, as illustrated by the following quotation: -

"We have a lot of people with renal failure, pneumonias and things, so for example, some of the [medicines that are] not continued, we identified as being nephrotoxic so we would not continue the medication". (Doctor, 2015)

Other patient characteristics included the age of the patient and level of frailty, particularly in terms of a dose being perceived to be too high or if a patient would no longer benefit from continuation of medicines, or any further additions.

\subsection{Drug characteristics}

In general, the underlying mantra of 'first do no harm' was seen as an important aspect of prescribing. For example, both doctors and pharmacists aimed to avoid harm by identifying side-effects or unwanted effects 
and were aware that polypharmacy could be detrimental.

Facilitator: "What are you seeking to achieve when you assess medication when you first see a patient?" "Basically - what they are taking and what they are supposed to be taking?" (Doctor A, 2017) "Are any of the medications causing the patient symptoms? Is there polypharmacy?" (Doctor B, 2017) "Are we worried about any side-effects?... the reason that the patient is here is because of the medications [that] can possibly [be] driving them." (Doctor C, 2017)

Knowledge of national or local guidelines influenced prescribers' ability to manage this as well as using them as justification for initiating prescribing. However, guidelines also limited individuals' prescribing independence with doctors indicating that they were reluctant to change an existing medicine (i.e. one that the patient had been taking prior to admission) unless overt clinical harm had occurred, or prompted by a specific guideline regardless of patient benefit profile.

These decisions were further modified according to their perceived knowledge of the pharmacological group of the drug. There was a reluctance to stop potential 'culprit' medicines because of a lack of knowledge, either of the drug itself, or the original reason for prescribing it. For example, "well generally, how long have they been on it? ... Let's be honest, we don't know how they work and also [I would be concerned about] the side effects of acute withdrawal..." (Doctor, 2016). "Yeah, and the exact rationale in terms of their use is not necessarily clear..."(Doctor, 2016). This appeared to apply particularly to medicines prescribed for psychiatric conditions. By contrast, participants accepted that they preferred to adjust familiar drugs with effects they believed were more predictable such as antihypertensive medications.

\subsection{Pharmacist factors}

Decisions to prescribe were thought to have been enhanced when pharmacists were involved. Doctors habitually seek advice from pharmacists present on the ward to assist in making prescribing decisions. Surprisingly, the pharmacists themselves appeared to be unaware of the high esteem within which they were held in terms of their potential ability to influence prescribing decisions. They tended to describe their role as being supportive and more as a "checker" or safety-net provider. Moreover, the pharmacists tended to compare themselves favourably with their medical colleagues with regard to performing medication reconciliation. The latter is the formal process, described in a toolkit published by the Royal Pharmaceutical Society ${ }^{19}$, that ensures accurate and complete medication information is obtained at interfaces of care such as when a patient is admitted to and discharged from hospital.

Facilitator (to pharmacists): "When assessing medication on admission, what are you seeking to achieve?" "... I would take an accurate drug history and reconcile that with the meds that have been prescribed. If there are any discrepancies, highlight them to the doctors... [and] highlight any incorrect doses as well to the doctors. Just make sure the basic safety is there and it matches with the patient that is there"(Pharmacist ). .." Also check allergies or anything that the doctors might not pick up such as eyedrops, creams, ointments, injections. (Pharmacist ) In response to a medication history documented in one of the case studies, one of the pharmacists commented: -I think ... the drug history has probably been done by a doctor... [there are] a lot of things we would have picked up like apixaban, codeine - we wouldn't just write '15-30mg prn'. We would write 'qds prn'. ... Then stuff like furosemide - we wouldn't write 'bd'. We would write 8am and 2pm. ... Movicol - we wouldn't write 'bd', we would write how many sachets they are having"(Pharmacist).

Doctors acknowledged that discharge prescriptions may be prepared based upon recommendations from pharmacists, as illustrated here: -

"I think, most of the time, when we are reviewing the medication at the time of discharge, we do the 'lifestyle', and then we go through the medication they came in with - [and we check to see] if the pharmacists are concerned about anything [by pharmacist highlighting in the notes]... (Doctor A, 2016). "If you have a really good pharmacist (Doctor B, 2016). "Yeah" (Doctor C, 2016). "If the pharmacist hasn't highlighted it, then actually they go home without those medications (Doctor D, 2016). 
The pharmacists did not always appreciate the clinical reasoning that underpinned prescribing decisions when an alternative action would be more obvious to them:-

Pharmacist 1: "Why are they on digoxin? Maybe they have developed AF [atrial fibrillation]?" Pharmacist 2: "Yeah", Pharmacist 3: "They are on bisoprolol 2.5mg, why don't they just optimise that [bisoprolol] first before starting digoxin?"Pharmacist 2: "They've got the patient on senna [which] can actually cause hypokalaemia which can cause digoxin toxicity".Pharmacist 1: "You would want to know if they are having palpitations".

The full potential of pharmacists with therapeutic knowledge was not evident within the doctors' focus group transcripts, a finding that has also been reported by Anderson et al. ${ }^{20}$ The skills of pharmacists appeared to be under-utilised with regard to new product prescribing, as has been reported by Tan et al. ${ }^{21}$ and in community pharmacy practice ${ }^{22}$, as well as a lack of formal partnership between the two groups resulting in the actions of pharmacists' being curtailed. Recent research in the UK demonstrates, however, that community pharmacists can conduct medication reviews and work effectively within a multidisciplinary team to tackle polypharmacy resulting in reduced re-admission rates after discharge from hospital. ${ }^{23} 24$

3.4 TrustworthinessThe concept of trustworthiness was relevant in terms of the veracity of medication records and, on a human level, between doctors themselves and their colleagues. Prescribing decisions reflected decision-making by doctors independently and in collaboration with pharmacists.

The way trustworthiness informed prescribing decisions was evident within the discourse of both doctors and pharmacists. On a 'systems' level, trustworthiness was apparent in terms of a perceived lack of reliability of the medication history and its currency in relation to the patient's presenting condition. Drug histories were viewed with a healthy degree of scepticism even when taken directly from patients. For example- "I ask them: 'Are you taking your medication'?" (Doctor 2, 2017)... "and they all say "yes"!' (Doctor 5, 2017). This was modified by the extent to which they thought patients were adhering to prescribed regimens. On a human level, there was evidence that some of the more experienced doctors did not always trust junior colleagues as active decision-makers - "It is usually the junior doctors who access the system and make the changes on senior instructions - so on the system it may look like the juniors are making decisions but they are just acting on instructions" (Doctor, 2015). Pharmacists, regardless of their level, were trusted by doctors to be more accurate regarding the recording of drug histories. This perception was reinforced by some pharmacists, who also believed there was degree of reliance upon them to pick up problems.

\subsection{Reliability of medication history}

Both doctors and pharmacists were cognisant of the information vacuum within which they were working at the AMU. The initial clerking of a patient focused upon obtaining up-to-date knowledge of the medication history and this was often thwarted because of incompleteness or datedness of records or lack of confidence in the patient's story. This was recognised particularly as a problem of prescribing within the unit as prescribers were working within an imperfect communications environment, piecing together a jigsaw, and filling in gaps using collateral sources. The following quotation is illustrative of frustration that several doctors expressed:

"The whole med rec [reconciliation] part of it is a nightmare in terms of ... getting access to what they are on reliably, and you can say - "yes" ["it is correct"]- not on a scrap bit of paper that they have written their drug doses down on 10 years before.... (Doctor 2016)

Owing to difficulties when working within an uncertain medication history, problems persist as prescribers' intentions remain insecure. For example,

"There [is] some medication [that is] specifically very distinctly difficult such as warfarin... you never know what the dose is and if the patient doesn't know then it's very difficult to prescribe appropriately. And also, insulin and pain relief - if it's 'prn' [when required] you may prescribe the lowest dose prn but they might be on the maximum dose which makes it very difficult(Doctor, 2015). "Sometimes... the medical notes don't match up to the EPMA [Electronic Prescribing and Medicines Administration] system... certain medicines 
have been missed off, so we're not too sure whether they have actually been stopped or just accidentally missed off" (Doctor, 2016).

Under such circumstances, it is clear that some prescribing decisions were left unmade with prescribers hoping that the issue will be picked up at a later point when more information or advice was available.

\subsection{Competing pressures and priorities}

The narrative of doctors illustrated that prescribing behaviour is contextualised by the environment within which they work. For example, owing to the limited number of doctors and reduced availability of pharmacists when working out of hours, a process of prioritisation arose by necessity.

"If it's 2'oclock in the morning... if you have other things going on, then you would prescribe the stuff that needs to be done and then highlight [other prescribing] to the day team". (Doctor, 2016)Sometimes, high workload levels mitigate against doctors responding to system alerts designed to flag up medication warnings which may be over-ridden during busy times. "... because you're doing a busy ward round /and a pop-up message arrives] you just press okay, okay. It's the automatic alerts that come up.... For pretty much everything and sometimes you say 'okay, carry on' [and] every click takes just 10 seconds" (Doctor, 2016).

One doctor recalled that he had been requested to expedite preparation of a 'take home' prescription for a patient - "... the review of the medications as a whole comes when the patient is in the discharge lounge and the nurses are on your neck - 'Do the TTO! Do the TTO! Do the TTO! So, you don't actually have time to review" (Doctor 2017).

\subsection{Responsibilities of prescribers}

There was evidence that prescribers develop demarcation lines whereby they identify where their responsibilities should be prioritised and, conversely, where tasks can be entrusted to others. This was apparent in the context of considering the extent to which the GP was responsible for the review of medication initiated pre-admission - "the GP is better placed at optimising those kind of doses unless we [believe we should] outright stop it. For optimisation we have to have good reason as to why we have chosen to optimise it. We only see the patient acutely" (Doctor, 2017).

The sentiments described in this theme overlap with the theme of competing priorities.

\subsection{Strengths and Limitations}

The strength of this study resides within the premise that it seeks to understand the mindset, in the real world, of both doctors and pharmacists, with varying levels of experience, associated with prescribing decisions on the AMU. The choice of an acute medical unit as the prescribing forum was advantageous because an admission to hospital presents an opportunity to review medication with potential to influence future prescribing decisions by the GP in the community. However, the study has a number of limitations including the fact that it was carried out at a single site, with a series of common presentations which may not, therefore, be representative of all prescribing situations, or of all AMUs throughout the UK. The focus group was conducted retrospectively, at least one day after the actual decisions had been made and therefore did not represent 'real-time' thought processes at the moment of prescribing or the thoughts of the actual prescriber.

Further research is needed that would ideally reflect the individual thought processes of prescribers and advisors prospectively at the time decisions are made and in samples of patients with a wider variety of diagnoses and in different acute hospital settings.

\subsection{Implications for practice}

An important factor identified in the present research was the perceived dearth of trusted medication history resulting in doctors understandably lacking confidence in making prescribing decisions based upon absent or inaccurate data. An inadequate medication history represents a significant shortcoming in the context of encouraging safe prescribing. Electronic patient medication records (ePMR) with alert systems are now 
routinely used in pharmacies and act as a safety-net prior to dispensing in pharmacies. Although ePMR are effective in alerting users of potential clinical hazards and errors, problems of false alerts and inconsistencies in alert management still persist. ${ }^{25}$ There remains, therefore, an on-going need to sit with the patient and take a traditional drug history to ensure not only prescribed medicines are included but also that alternative therapies, treatments or lifestyle medicines that have been purchased from pharmacies, or bought over the internet, are also included. The perceived value of pharmacists, as experts in taking a medication history, lends support to an argument for pharmacists to be present on the AMU out of hours in order to provide support for medical staff in the medicines reconciliation process.

Pressure from patients, relatives or carers may result in uncomfortable prescribing decisions by hospital prescribers, ${ }^{26}$ but stress within the work environment has not yet been acknowledged in relation to prescribing decisions. The finding that there are competing priorities encountered by doctors on the AMU implies that it is unrealistic to assume an effective medication review can be completed immediately prior to a patient being discharged. A suggested way forward would be to allocate staff with protected time to overcome these challenges. Alternatively, proven schemes could be expanded, such as those in Scotland ${ }^{23}$ and Cornwall ${ }^{24}$, where the value of community pharmacists reviewing medicines of patients who have recently been discharged from hospital has been demonstrated. The AMU is recognised as being a high-pressure working environment owing to the clinical urgency of patients' admissions as well as the need to maintain patient flow through the hospital. If doctors feel under pressure, they may focus principally on the primary diagnosis, prescribing medicines that are efficacious in relation to symptoms associated with the cause of admission rather than to plan the discharge prescription in the context of avoiding longer-term inappropriate polypharmacy. Such an approach may ultimately increase the likelihood of patients experiencing an adverse drug event in primary care as outlined by Slight et al. ${ }^{27}$ Moreover, perceived workplace pressure may legitimise hospital prescribers, underpinned by recent UK guidance to assign accountability to the GP for the continuation or modification of medicines that were not initiated in hospital. ${ }^{28}$

Prescribing tools such as Beers and STOPP/START criteria have been available for over a decade and have been integrated into computerised clinical decision support systems (CDSS). Prescribing tools have been successfully deployed to quantify the incidence of inappropriate prescribing but several studies over recent years, in a variety of clinical settings, have demonstrated that there is insufficient evidence of their clinical or economic impact. ${ }^{14,29,30,31,32,33}$ The failure of prescribing tools such as STOPP/START to reduce the incidence of inappropriate prescribing suggests that an alternative approach may be worthy of consideration. Hence, we propose that a greater understanding of factors that directly influence doctors during routine practice at the point of prescribing may help point the way towards providing better support for prescribers, including CDSS support, to ensure prescribing guidance translates into patient benefit.

\section{REFERENCES}

1. Allan F, Guthrie B, Kelman A. Polypharmacy: a framework for theory and practice. Pharm J 2019 303: 324-331. https://www.pharmaceutical-journal.com/cpd-and-learning/cpd-article/polypharmacya-framework-for-theory-and-practice/20207246.cpdarticle?

2. Pirmohamed M, James S, Meakin S et al. Adverse drug reactions as cause of admission to hospital: prospective analysis of 18,820 patients. BMJ. 2004 329:15-19 https://doi:10.1136/bmj.329.7456.15.

3. Multimorbidity and polypharmacy. NICE key therapeutics topic (KTT18). 2017. : https://www.nice.org.uk/advice/ktt18. Accessed July 2020.

4. Murshid MA and Mohaidin Z. Models and theories of prescribing decisions: A review and suggested new model. Pharm Pract (Granada) 201715 2:990. https://doi:10.18549/PharmPract.2017.02.990

5. Stros $M$ and Lee N. Marketing dimensions in the prescription pharmaceutical industry: a systematic literature review. J Strategic Mark. 2015;23 (4):318-336. https://doi.org/10.1080/0965254X.2014.931878. Accessed July 2020.

6. Kyle GJ et al. Pharmaceutical company influences on medication prescribing and their potential impact on quality use of medicines. J Clin Pharm Ther 2008;33(5):553-559. https://doi.org/10.1111/j.13652710.2008.00948.x. Accessed July 2020. 
7. A patient-centred approach to polypharmacy. NHS Specialist Pharmacy Service. 2017. : https://www.sps.nhs.uk/articles/polypharmacy-oligopharmacy-deprescribing-resources-to-supportlocal-delivery/ Accessed January 4, 2020.

8. Duerden $\mathrm{M}$ et al. Polypharmacy and medicines optimisation: making it safe and sound. The King's Fund. 2013. : https://www.kingsfund.org.uk/sites/default/files/field/field_publication_file/polypharmacy-and-medicines-optimisation-kingsfund-nov13.pdf. Accessed July 2020.

9. Royal Pharmaceutical Society. Polypharmacy: Getting our medicines right. 2019. https://www.rpharms.com/recognition/setting-professional-standards/polypharmacy-getting-ourmedicines-right. Accessed July 2020.

10. Polypharmacy guidance: realistic prescribing. $3^{\text {rd } E d n . ~ 2018 . ~ S c o t t i s h ~ g o v e r n m e n t . ~: ~}$ https://www.therapeutics.scot.nhs.uk/wp-content/uploads/2018/04/Polypharmacy-Guidance2018.pdf. Accessed July 2020.

11. Polypharmacy: guidance for prescribing. All Wales Medicines Strategy Group. 2014 . : http://awmsg.org/docs/awmsg/medman/Polypharmacy\%20-\%20Guidance\%20for\%20Prescribing.pdf. Accessed July 2020.

12. American Geriatrics Society Beers criteria Update Expert Panel. American Geriatrics Society 2019 updated AGS Beers criteria for potentially inappropriate medication use in older adults. J Am Geriatr Soc 2019:67(4):674-694. https://doi.org/10.1111/jgs.15767.

13. O'Mahony D, O'Sullivan D, Byrne S, O'Connor MN, Ryan C, Gallagher G. STOPP/START criteria for potentially inappropriate prescribing in older people: version 2: Age Ageing 2015;44 (2): 213-218. https://doi.org/10.1093/ageing/afu145

14. O'Mahony D, Gudmundsson A, Soiza RL, et al. Prevention of adverse reactions in hospitalized older patients with multi-morbidity and polypharmacy: the SENATOR randomized controlled trial. Age Ageing 2020;1 https://doi.org/10.1093/ageing/afaa072.

15. Aronson JA pragmatic view of thematic analysis 1995. The Qualitative Report, 2 (1), 1-3. https://nsuworks.edu/tqr/vol2/iss1/3.

16. Outhwaite W.1975. Understanding Social Life: The Method Called Verstehen. London: George Allen Unwin.

17. Symon G \& Cassell C (2012). Qualitative Organizational Research: Core Methods and Current Challenges. Sage, London. $20 \mathrm{p}$.

18. Patwardhan AR. Physicians-Pharmaceutical Sales Representatives Interactions and Conflict of Interest: Challenges and Solutions. The Journal of Health Care Organization, Provision, and Financing 2016 53: 1-5. https://doi.org/10.1177/0046958016667597.

19. Royal Pharmaceutical Society 2015. Medicines Reconciliation: Best practice Resource and Toolkit. https://www.sps.nhs.uk/articles/medicines-reconciliation-best-practice-resource-and-toolkit. Accessed July 2020.

20. Anderson $\mathrm{C}$ et al. Feedback from community pharmacy users on the contribution of community pharmacy to improving the public's health: a systematic review of the peer reviewed and non-peer reviewed literature 1990-2002. Health Expect 20047 (3):191-202. https://doi.org/10.1111/j.13697625.2004.00274.x

21. Tan ECK, Stewart K, Elliott RA, Johnson G. Pharmacist consultation in general practice clinics: The Pharmacists in Practice Study. Res Social Adm Pharm 2014 10: 623-632. https://doi.org/10.1016/j.sapharm.2013.08.005.

22. Rivers PH, Waterfield J, Grootveld M. Exploring the prevalence of and factors associated with advice on prescription medicines: a survey of community pharmacies in an English city. Health Soc Care Comm 2017:25(6):1774-1786. https://doi.org/10.1111/hsc.12451.

23. Din R, Sardar CM, Bryson G, McGarry V. Evaluation of a pharmacist-led polypharmacy review project 2020. Pharm J 304: 340-347. https://doi.org/10.1211/PJ.2020.20207920.

24. Wilcock M, Sibley A, Blackwell R, Kluettgens B, Robens S, Bastian L. Involving community pharmacists in transfer of care from hospital: Indications of a reduced 30-day hospital readmission rates for patients in Cornwall. Int.J Pharm Prac 202028 (1). https://doi.org/10.1111/ijpp.12603. 
25. Olowagbemileke O, Avery A, Gupta A, Boyd M. The evidence for the effectiveness of safety alerts in electronic patient medication record systems at the point of pharmacy order entry: a systematic review. BMC Med Inform Decis 201313: 69. http://www.biomedcentral.com/1472-6947/13/69.

26. Lewis PJ, Tully MP. The discomfort caused by patient pressure on the prescribing decisions of hospital prescribers 2011 Res Soc Adm Pharm 7: 4-15. https://doi.org/10.1258/jrsm.2009.090150.

27. Slight SP, Howard R, Ghaleb M et al.The causes of prescribing errors in English general practices: a qualitative study Br J Gen Pract 2013; 63 (615) e713-e720. https://doi.org/10.3399/bjgp13X673739.

28. Primary Care Delivery: Policy \& Strategy, Operations \& Information Directorate NHS England Responsibility for prescribing between Primary \& Secondary/Tertiary Care: January 2018: : https://www.england.nhs.uk/wp-content/uploads/2018/03/responsibility-prescribingbetween-primary-secondary-care-v2.pdf). Accessed July 2020.

29. Hill-Taylor B, Sketris I, Hayden J, Byrne S, OSullivan D, Christie R. Application of STOPP/START criteria: a systematic review of the prevalence of inappropriate prescribing in older adults, and evidence of clinical, humanistic and economic impact. J Clin Pharm Ther 201338 (5):360-372. https://doi.org/10.1111/jcpt.12059.

30. Verdoorn S, Kwint HF, Hoogland P, Gussekloo MD, Bouvy ML. Drug related problems identified during medication review before and after the introduction of a clinical decision support system. J Clin Pharm Ther 2017 43: 224 - 231. https://doi.org/10.1111/jcpt.12637.

31. Meulendijk MC, Spruit MR, Drenth-van Maanen AC et al. Computerized decision support improves medication review effectiveness: an experiment evaluating the STRIP Assistant's usability. Drugs Aging 2015 32: 495-503. https://doi.org/10.1007/s40266-015-0270-0

32. Monteiro C, Canario C, Ribeiro MA, Duarte AP, Alves G. Medication evaluation in Portuguese elderly patients according to Beers, STOPP/START criteria and EU (7)-PIM List - An Exploratory Study 2020 Patient Prefer Adher 14: 795-802. doi:10.2147/PPA.S247013.

33. Thomas RE. Assessing medication problems in those $>65$ using the STOPP and START criteria. Current Aging Science 2016 9: 150-159 http://doi.org/10.2174/1874609809999160217101716.

\section{ACKNOWLEDGEMENTS}

The authors gratefully acknowledge the contribution of the following who, as students, were involved in this research when undertaking their final year MPharm projects: Iman Ahmed, Mobeen Aziz, Kaash Chandarana, Jignasa Chawda, Navpreet Gill, Kiran Hothi, Mohsin Patel, Ramanpreet Rai.

\section{CONFLICT OF INTEREST}

The authors declare no potential conflicts of interest with respect to the research, authorship, and / or publication of this article.

Table 1: Subthemes relating to prescribing decisions identified from data analysis ${ }^{1}$ and existing prescribing decision theory ${ }^{2}$

\begin{tabular}{ll}
\hline DIMENSION & SUBTHEME \\
\hline Patient characteristics $^{1,2}$ & Presenting complaint or pathology \\
& Age of patient \\
& Wishes of family \\
& Adherence \\
& Clinical picture informing prescribing decisions \\
& Avoiding harm \\
& Polypharmacy \\
& Drawing upon guidelines as resource (e.g. NICE) \\
& Iatrogenesis \\
& Review / lack of review of medication \\
& Pharmacist as checker and optimiser, not clinical \\
& decision maker
\end{tabular}


DIMENSION

Trustworthiness ${ }^{1,2}$

Marketing effects ${ }^{2}$

Reliability of medication history (written and verbal) ${ }^{1}$

External pressures \& priorities $^{1}$

Responsibilities of prescribers ${ }^{1}$
SUBTHEME

Expertise

Distrust of information source until proved correct

/ reconciled

Doctors compared with pharmacists and

vice-versa

Perception that junior doctors may not have enough experience to review drugs

Not overtly observed in verbatim transcripts

Confidence in obtaining accurate history Filling in

knowledge gaps from collateral sources Availability

\& functionality of Electronic Prescribing \&

Administration System / use of decision support tools Language ambiguity

Time-consuming aspects of job

Pressure to expedite 'take home' prescription on discharge

Coping strategies in response to demands on workload

Reluctance to change medicines perceived not to be associated with cause of admission

Passing the buck. Perception that GP (not self) is responsible for prescribing drugs that were

originated in primary care setting 